\title{
Desensitization for Allergic Reactions to Chemotherapy
}

\author{
David In-Chull Hong \\ Division of Rheumatology, Immunology and Allergy, Brigham \& Women's Hospital, Boston, MA, USA.
}

\begin{abstract}
Drug desensitization is procedure by which patients can be tolerized to medications that have previously induced hypersensitivity reactions. Initially used in treating hypersensitivity reactions to antibiotics, desensitization is now frequently used in the setting of allergy to chemotherapy drugs and biologics, thus widening the clinical applicability of this procedure which has been proven to be quite safe and effective in improving clinical outcomes, mainly by allowing patients to remain on preferred first-line therapy. This paper reviews the history, application, and safety studies of drug desensitization for chemotherapy and biologics.
\end{abstract}

Key Words: Drug hypsersensitivity, desensitization, anaphylaxis

\section{INTRODUCTION}

True drug allergy is an immune-mediated adverse drug reaction that can be a serious impediment to treating patients with first-line or preferred therapy. In many cases, there is a suitable alternative, but there are some clinical situations where a particular drug is clearly the superior or only option. While delayed-onset drug rashes tend to be the most common type of drug allergy reaction, they are usually only cutaneous whereas immediate onset anaphylactic reactions can involve multiple organ systems and typically occur in the setting of infusions. Anaphylaxis is caused by release of preformed and rapidly-formed mediators (i.e., histamine, leukotrienes, prostaglandins) from mast cells and basophils resulting in symptoms of urticaria, flushing, hypotension, dyspnea/hypoxia, and/or nausea/severe GI disturbance. ${ }^{1}$ Cardiopulmonary anaphylaxis is potentially life-threatening, especially in patients who suffer from chronic disease diminishing their cardiopulmonary reserve (i.e., asthma, severe COPD, heart failure). Usually, such reactions necessitate strict avoidance going forward. In many settings, low-grade hypersensitivity

Received: October 12, 2018

Corresponding author: David In-Chull Hong, MD, Division of Rheumatology, Immunology and Allergy, Brigham \& Women's Hospital, 60 Fenwood Rd., Boston, MA 02115 USA.

Tel: 1-617-732-1300, Fax: 1-617-731-2748, E-mail: dhong@bwh.harvard.edu

-The author has no potential conflicts of interest to disclose.

(C) Copyright: Yonsei University College of Medicine 2019

This is an Open Access article distributed under the terms of the Creative Commons Attribution Non-Commercial License (https://creativecommons.org/licenses/ by-nc/4.0) which permits unrestricted non-commercial use, distribution, and reproduction in any medium, provided the original work is properly cited. reactions are treated with slowing down the rate of infusion and increasing the doses and/or varieties of premedications (i.e., steroids, antihistamines, leukotriene receptor antagonists). Avoidance is usually recommended for higher grade anaphylaxis. ${ }^{2}$ A commonly used anaphylaxis grading system by Brown, et al. ${ }^{3}$ categorizes grade 1 anaphylaxis as immediate onset symptoms of a strictly cutaneous nature. Grade 2 reactions involve subjective symptoms suggestive of organ system involvement (dyspnea, stridor, wheeze, pre-scyncope, throat tightness). Grade 3 anaphylaxis features severe symptoms such as syncope, incontinence, and/or objective vital sign derangements such as hypotension or hypoxia in the setting of correlative symptoms. ${ }^{3}$ For those clinical situations where high grade anaphylaxis prevents the administration of a critically necessary drug, drug desensitization can be an extremely useful addition to the clinical toolkit.

Rapid drug desensitization was first successfully accomplished in the 1940s to penicillins for patients with infections requiring their use. ${ }^{4,5}$ It has since then been applied to other antibiotic families including carbapenems ${ }^{6}$, cephalosporins ${ }^{7}$, and quinolones. ${ }^{8}$ Drug desensitization is a procedure by which an offending drug is administered over a series of very gradual dose increments such that the sum total dose equals the original target dose of the drug. The exact mechanism by which desensitization works has yet to be fully elucidated, but in most cases, it markedly reduces or completely abrogates hypersensitivity symptoms compared to initial presentation. Usually, premedications that antagonize the effect of mediators of immediate hypersensitivity (histamine, leukotrienes, prostaglandins) are often given to lessen the risk of breakthrough symptoms which may occur during the course of a 
desensitization. While drug desensitization is useful for treating anaphylactic drug reactions, it is not useful in the setting of Stevens Johnson, severe exfoliative dermatitis, drug reaction with eosinophilia and systemic symptoms (DRESS syndrome), or acute generalized exanthemetous pustulosis (AGEP). Its use in delayed-onset drug rash is also controversial and likely not useful since desensitization mainly targets cells involved in immediate hypersensitivity reactions (i.e., mast cells and basophils).

Over the past 15 years, drug desensitization has found important application in treating chemotherapy allergy which can have significant implications for patients who may have limited options to treat their malignancy. While it may be possible to treat through severe infusion reactions with high-dose antihistamines and steroids, if this approach fails the only remaining option is to switch to second or third line therapy, assuming a suitable alternative even exists. Typically, non-first line agents are less efficacious and/or have more side effects.

\section{DESENSITIZATION FOR CHEMOTHERAPY}

In the early 2000s, multiple clinical trials by the Gynecologic Oncology Group established the combination of carboplatin and paclitaxel to be superior and less toxic than other regimens for the treatment of high-grade ovarian cancer. ${ }^{9,10}$ Around the same time, it was becoming recognized that one of the complications of this treatment regimen was the relatively high prevalence of hypersensitivity reactions to carboplatin in women who received repeated courses of chemotherapy. Ovarian cancer is a silent cancer which is often discovered at a relatively advanced stage with large or microscopic metastases anywhere within the peritoneal cavity. As a result, many women with this cancer require repeated courses of chemotherapy. ${ }^{11}$ Studies revealed that on average, about $12 \%$ of patients who received 8 or more lifetime infusions of carboplatin will develop a hypersensitivity reaction to this drug with positive skin testing, meaning this is an acquired hypersensitivity from sensitization. ${ }^{12,13}$ The incidence of carboplatin allergy may also increase with continuing exposure to carboplatin beyond 8 cycles. ${ }^{14}$ Skin testing appears to be a useful risk stratification tool for the assessment of carboplatin allergy, and may help providers determine which patients need desensitization versus those who do not. ${ }^{15}$ Additionally, $30 \%$ of patients who received Taxol will experience severe acute infusion reactions as well. ${ }^{16,17}$ As a result of this new treatment standard for ovarian cancer, an increasing number of patients were being referred to the Allergy Clinic at Brigham and Women's Hospital (BWH). Many of these allergic reactions were consistent with anaphylaxis of varying grades, and most patients referred were positive on skin testing to carboplatin. Because these reactions were still consistent with immediate type hypersensitivity, the possibility of desensitizing these patients was considered.
From 2002 through 2004, Lee, et al. ${ }^{18}$ successfully desensitized 10 patients using a protocol in which the chemotherapy was administered over 12 discrete steps using 3 separate solutions of carboplatin of varying concentration (Fig. 1). In this initial report, 10 patients completed 35 courses of carboplatin desensitizations, of which 31 were completed with no reactions. The remaining 4 desensitizations were complicated by mild cutaneous reactions which did not prevent completion of the infusions. The chemotherapy drug desensitization program was expanded, and in 2008, Castells, et al. ${ }^{19}$ published a case series of 98 patients who underwent 413 desensitizations. Ninety-four percent of the desensitizations performed had no reactions or very mild strictly cutaneous reactions that responded quickly to extra antihistamine. The list of drugs successfully desensitized to was later expanded to include other chemotherapy drugs such as paclitaxel, ${ }^{20}$ cisplatin, oxaliplatin, and doxorubicin. ${ }^{19}$ Eventually, monoclonal agents such as rituximab, infliximab, trastuzumab, and bevicizumab were desensitized to, thereby expanding the therapeutic reach of desensitizations to other disease entities such as seropositive arthritis, inflammatory bowel disease, and uveitis. ${ }^{21}$ Unlike carboplatin, most reactions to non-platin chemotherapy drugs and monoclonals were not classical for type I IgE-mediated reactions since many reactions occurred with the 1st or 2nd exposure. Skin testing to non-platin chemotherapy drugs also had unclear sensitivity or specificity, but reactions would typically respond to stopping the infusion and administering antihistamines and steroids. Symptoms of flushing, hypotension, warmth, pruritus, and dyspnea were furthermore classic for anaphylaxis despite the lack of positive skin test findings. Given the clinical history, patients were empirically desensitized using the same 3-solution protocol as for carboplatin with similar rates of success.

\section{NON-IgE-MEDIATED ANAPHYLACTIC REACTIONS}

Aside from platin-based chemotherapy drugs, many other drugs which cause acute infusion reactions probably do so in an IgE-independent manner since many reactions occur with the first or second exposure before a patient could theoretically be sensitized with drug-specific IgE. How and why this happens is a subject of interest and reveals other mechanisms of drug hypersensitivity.

Paclitaxel is a chemotherapy drug derived from the needles or bark of yew trees that requires solubilization into aqueous solutions with polyethoxyated castor oil, also known under the brand-name Cremaphor. Cremaphor can cause direct histamine release from mast cells and is thought to be the main cause of non-IgE-mediated anaphylactic reactions in patients who have reactions to Taxol. ${ }^{22}$ Reactions to Cremaphor include flushing, urticaria, back pain, chest tightness, 


\begin{tabular}{l|r} 
Name of medication: & \multicolumn{2}{c}{ carboplatin } \\
\cline { 2 - 2 } Target Dose $(\mathrm{mg})$ & 150 \\
Standard volume per bag $(\mathrm{ml})$ & 250 \\
Final rate of infusion $(\mathrm{ml} / \mathrm{hr})$ & 80 \\
& \\
Calculated target concentration $(\mathrm{mg} / \mathrm{ml})$ & 0.6 \\
Standard time of infusion (minutes) & 187.5
\end{tabular}

\begin{tabular}{|c|c|c|c|c|c|}
\hline \multirow[b]{3}{*}{ Solution 1} & \multirow[b]{3}{*}{250} & \multirow[b]{3}{*}{$\mathrm{ml}$ of } & \multirow[b]{3}{*}{$0.006 \mathrm{mg} / \mathrm{ml}$} & \multicolumn{2}{|r|}{ Amount of bag infused } \\
\hline & & & & Total mg per bag & $(\mathrm{ml})$ \\
\hline & & & & 1.500 & 9.25 \\
\hline Solution 2 & 250 & $\mathrm{ml}$ of & $0.060 \mathrm{mg} / \mathrm{ml}$ & 15.000 & 18.75 \\
\hline Solution 3 & 250 & $\mathrm{ml}$ of & $0.595 \mathrm{mg} / \mathrm{ml}$ & 148.820 & 250.00 \\
\hline
\end{tabular}

\begin{tabular}{cccccrr} 
Step & Solution & Rate (ml/hr) & Time (min) & $\begin{array}{c}\text { Volume } \\
\text { infused per } \\
\text { step (ml) }\end{array}$ & $\begin{array}{r}\text { Dose administered } \\
\text { with this step }(\mathbf{m g})\end{array}$ & $\begin{array}{r}\text { Cumulative dose } \\
(\mathbf{m g})\end{array}$ \\
\hline 1 & 1 & 2.0 & 15 & 0.50 & 0.0030 & 0.0030 \\
2 & 1 & 5.0 & 15 & 1.25 & 0.0075 & 0.0105 \\
3 & 1 & 10.0 & 15 & 2.50 & 0.0150 & 0.0255 \\
4 & 1 & 20.0 & 15 & 5.00 & 0.0300 & 0.0555 \\
\hline 5 & 2 & 5.0 & 15 & 1.25 & 0.0750 & 0.1305 \\
6 & 2 & 10.0 & 15 & 2.50 & 0.1500 & 0.2805 \\
7 & 2 & 20.0 & 15 & 5.00 & 0.3000 & 0.5805 \\
8 & 2 & 40.0 & 15 & 10.00 & 0.6000 & 1.1805 \\
\hline 9 & 3 & 10.0 & 15 & 2.50 & 1.4882 & 2.6687 \\
10 & 3 & 20.0 & 15 & 5.00 & 2.9764 & 5.6451 \\
11 & 3 & 40.0 & 15 & 10.00 & 5.9528 & 11.5979 \\
12 & 3 & 80.0 & 174.375 & 232.50 & 138.4021 & 150.0000 \\
\hline
\end{tabular}

Fig. 1. Example of a 3-bag, 12-step desensitization protocol for carboplatin.

and wheezing. Cremaphor is known to cause histamine release in $\operatorname{dogs}^{23}$ and may do so via production of anaphylotoxins secondary to activation of the classical complement pathway. ${ }^{24,25}$ Strong evidence that Cremaphor is the primary cause of reactions in Taxol-sensitive patients is demonstrated in the nonreactivity of the same patients when they are challenged with Abraxane which is paclitaxel encapsulated in albumin nanoparticles to solubilize into aqueous solutions. ${ }^{26}$ This formulation of paclitaxel contains no Cremaphor. Hypersensitivity reactions to Abraxane are extremely rare but the main barrier to access is the extremely high cost of this drug over Taxol.

Because reactions to Taxol are frequently non-IgE mediated, reactions commonly occur with the first or second lifetime infusion, unlike with carboplatin which requires multiple exposures to sensitize the patient with drug specific IgE. Although reactions to Taxol are frequently non-IgE mediated, patients typically respond well to desensitization. The utility of skin testing for Taxol reactions is somewhat limited by its uncertain specificity since some patients may have a pre-existing sensitivity to Taxol due to prior sensitization to a crossreactive plant protein. Thus it is possible that in a small subset of patients, allergy to Taxol represents an IgE-mediated process. One of the main arguments supporting the need for paclitaxel skin testing is in identifying patients who have infusion reactions but may not need desensitization. For example, Cremaphor-induced reactions may be idiosyncratic and not reproducible, while other cases may involve very mild or de- layed-onset reactions. Picard, et al. ${ }^{27}$ described a diagnostic algorithm to better define patients who need desensitization for taxanes versus those who did not using clinical history and skin testing as a risk stratification tool. In their study, 36 out of 164 patients with a history of hypersensitivity reaction to paclitaxel or docetaxel were able to return to regular infusion without desensitization using drug challenges.

Drug challenges, as opposed to drug desensitizations, are a method by which patients can be carefully monitored for allergic reactions by giving drug in small test doses before giving the remainder of the dose. There is no drug dilution involved with challenges, and the procedure is done to see whether a patient has hypersensitivity or not by trying to replicate actual infusion conditions after demonstration of tolerance to the small test doses. A negative challenge usually means the patient is not hypersensitive and helps to rule out drug allergy. Because challenges are the gold standard to rule out reproducible hypersensitivity reactions, they can be a useful tool to validate the positive predictive value of skin testing. Intentionally challenging a patient to something they are positive to on a skin test is not commonly done, but Markman, et al..$^{13}$ did just that in 7 patients with a positive skin test, 6 of whom had a reaction, thus giving carboplatin skin testing a positive predictive value of $86 \%$. To this author's knowledge, this is the only instance of such a study.

Whereas challenges ask the question of whether a patient is truly allergic or not, a drug desensitization assumes the pa- 
tient is allergic to the medication. Instead of giving test doses, desensitization dosing usually starts at a much smaller starting point and is uptitrated more gradually in smaller and more frequent increments. Patients in the Picard study ${ }^{27}$ who were challenged typically had delayed-onset or grade 1 reactions and also had negative skin testing, though there were also some patients with grade 2 reactions who were also able to successfully return to regular infusion without desensitization. In their diagnostic algorithm, patients with either grade 1 or 2 reactions who also had negative skin testing to paclitaxel or docetaxel were challenged using a 3-step protocol (Fig. 2).

Another inactive ingredient found in chemotherapy drugs is polysorbate 80 (PS80) which is found in another taxane drug, taxotere, as well as certain biologicals including infliximab and rituximab. PS80 is a surfactant which can also directly activate mast cells, most likely through the breakdown of PS80 into peroxide radicals. The generation of peroxide radicals in drugs containing PS80 may be enhanced with unnecessary exposure of the drug to heat and/or light. ${ }^{28}$

A curiously interesting example of how a drug may cause allergic reactions on first exposure is found in cetuximab, an anti-epidermal growth factor receptor (EGFR) monoclonal used in the treatment of colon cancer, lung cancer, and head and neck cancer. Cases of anaphylaxis to cetuximab were found to be secondary to the presence of IgE recognizing an oligosaccharide known as galactose-alpha-1,3-galactose, commonly referred to as alpha-gal. Anti-alpha-gal IgE has been implicated in cases of delayed-onset anaphylaxis induced by mammalian meat consumption thought to be conferred on patients as a result of tick bites since there is a higher prevalence of mammalian meat allergy in parts of the United States where a certain tick species, Amblyomma americanum, is found. ${ }^{29,30}$ The connection between meat allergy and cetuximab allergy was made when it was discovered that the cell line in which cetuximab is produced expresses a transferase that attaches the alpha-gal moiety onto the drug's heavy chain. ${ }^{31}$

\section{SAFETY AND EFFICACY}

Since the early 2000s, thousands of desensitizations were successfully completed at Dana-Farber Cancer Institute and BWH. A recent paper by Sloane, et al. ${ }^{32}$ corroborated safety and efficacy data from earlier papers using a larger data subset. From 2007 to 2010, 2177 desensitizations were completed on 370 patients with a nearly $100 \%$ completion rate. Nearly $100 \%$ of infusions were completed and 93\% (2023) were completed with no reaction (1605) or with simple mild cutaneous reactions (418) (Fig. 3). Agents desensitized to included traditional chemotherapy drugs (i.e., platins, paclitaxel, cyclophosphamide) and biologics (i.e., rituximab, infliximab, trastuzumab, bevacizumab). This paper also showed that desensitization was quite effective regardless of how severe the initial presenting rate of anaphylaxis was. As a result, it was discovered that most desensitizations could be performed in the outpatient setting, even for grade 3 anaphylactic reactions. This was significant in that most desensitizations were initially performed in the intensive care unit setting with a 1:1 nurse to patient ratio before patients could be moved to the outpatient setting. Because desensitization was found to

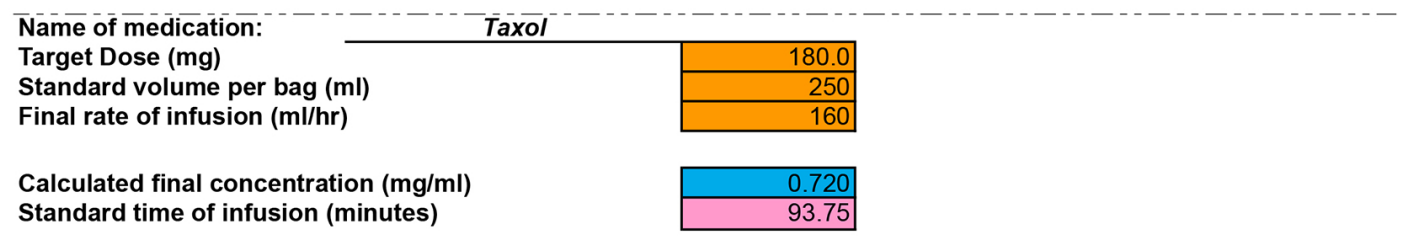

\begin{tabular}{l|l|l|r|r|r|r|r}
\multicolumn{3}{c}{ Total mg per bag Total vol used (mL) } \\
\hline Solution 1 & 250 & $\mathrm{ml}$ of & 0.720 & $\mathrm{mg} / \mathrm{ml}$ & 180.000 & 250.03 \\
\hline
\end{tabular}

${ }^{* * *}$ PLEASE NOTE *** The total volume and dose dispensed are more than the final dose given to patient because many of the solutions are not completely infused

\begin{tabular}{|c|c|c|c|c|c|c|}
\hline Step & Solution & Rate (ml/hr) & Time (min) & $\begin{array}{c}\text { Volume } \\
\text { infused per } \\
\text { step (ml) }\end{array}$ & $\begin{array}{l}\text { Dose administered } \\
\text { with this step (mg) }\end{array}$ & $\begin{array}{r}\text { Cumulative dose } \\
(\mathrm{mg}) \\
\end{array}$ \\
\hline 1 & 1 & 2.0 & 15 & 0.50 & 0.3600 & 0.3600 \\
\hline 2 & 1 & 20.0 & 15 & 5.00 & 3.6000 & 3.9600 \\
\hline 3 & 1 & 160.0 & 91.7 & 244.53 & 176.0640 & 180.0240 \\
\hline
\end{tabular}

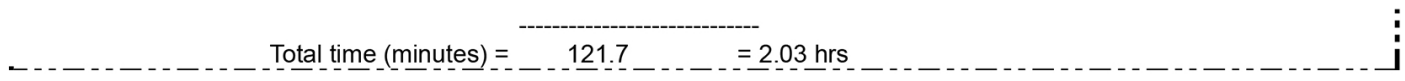

Fig. 2. Example of 1-bag, 3-step challenge protocol for Taxol. 

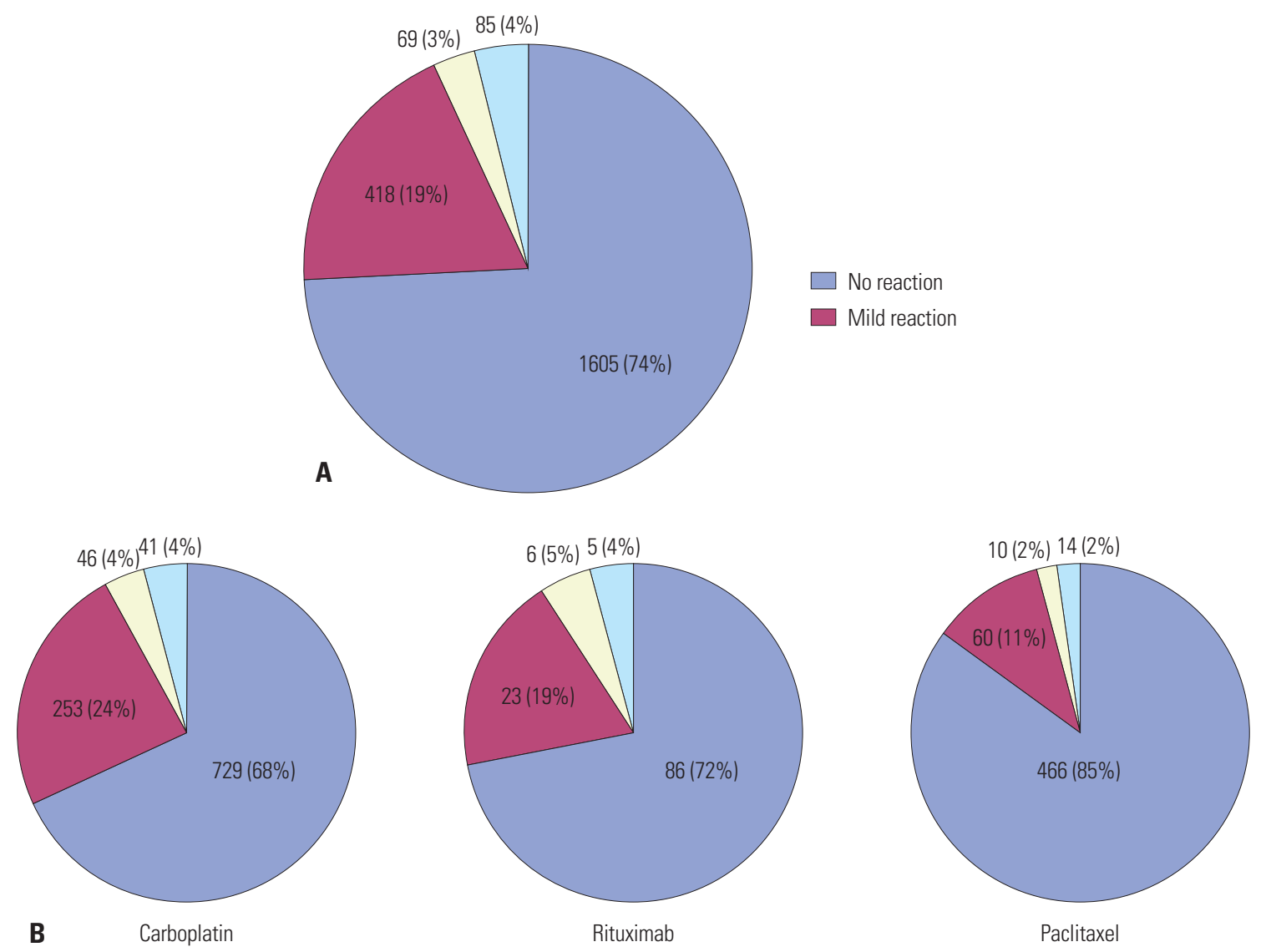

\begin{tabular}{|c|c|c|c|c|c|}
\hline \multirow{2}{*}{ Year } & \multicolumn{4}{|c|}{ Desensitization reaction grade } & \multirow{2}{*}{ Total } \\
\hline & 0 & 1 & 2 & 3 & \\
\hline 2007 & $236(66 \%)$ & $81(23 \%)$ & $18(5 \%)$ & $23(6 \%)$ & 358 \\
\hline 2008 & $341(67 \%)$ & $126(25 \%)$ & $20(4 \%)$ & $23(5 \%)$ & 510 \\
\hline 2009 & $434(77 \%)$ & $104(18 \%)$ & $15(3 \%)$ & $14(2 \%)$ & 567 \\
\hline 2010 & $594(80 \%)$ & $107(14 \%)$ & $16(2 \%)$ & $25(3 \%)$ & 742 \\
\hline Total & 1605 & 418 & 69 & 85 & 2177 \\
\hline
\end{tabular}

Fig. 3. Safety of desensitizations. (A) The overall number and severity of breakthrough reactions occurring during all desensitization from 2007 to 2010 at Brigham and Women's Hospital/Dana-Farber Cancer Institute is shown as a total of all desensitizations (2177) along with (B) specific data for carboplatin, rituximab, and paclitaxel, the most commonly desensitized drugs. (C) Breakdown of breakthrough reactions for all desensitizations. Adapted from Sloane, et al. J Allergy Clin Immunol Pract 2016;4:497-504, with permission of Elsevier [32]

be effective for both low and high-grade anaphylactic reactions, more initial desensitizations could be performed in the outpatient setting, thereby reducing the overall resource demands and healthcare costs for patients requiring desensitization. At BWH, we have had an outstanding safety record by making continuous quality and safety improvements in how the desensitization team operates such that many of our patients are routinely desensitized with a 1:2 nurse to patient ratio. 1:1 nursing is now the exception rather than the rule and has greatly increased our center's ability to accomodate patients who need chemotherapy desensitization. Scheduling desensitizations in the outpatient setting also gives patients a greater sense of control and certainty over the treatment since inpatient desensitization access may be limited by availability of inpatient resources.

Performing more desensitizations in the outpatient setting also increases access to patients who need it. While desensitization may require more resources than a typical infusion visit, Sloane, et al..$^{32}$ asked the question of whether desensitization might be cost-neutral by reducing morbidity and improving overall clinical outcomes as a result of keeping patients on primary first-line therapy. In their analysis, they found that patients who were being desensitized had roughly equivalent overall healthcare costs as patients who did not require desensitization because they had fewer admissions, unscheduled $\mathrm{MD}$ visits, and ER visits than patients who had to switch therapy because of their history of hypersensitivity reaction.

Another question asked by the group was whether chemo- 
therapy administered through desensitization was as efficacious as the same treatment given by regular infusion, potentially as a result of altered pharmacokinetics from the longer infusion and different concentrations administered. Using carboplatin desensitizations as a model to study non-inferiority, Sloane, et al. ${ }^{32}$ found a nonsignificant improvement in mortality on Kaplan-Meyer survival analysis between patients who were being desensitized and those who received the same chemotherapy by regular infusion. This suggests that at the least, chemotherapy administered by desensitization is not therapeutically inferior to regular infusion (Fig. 4).

\section{CONCLUSIONS}

Drug desensitization in the 21st century has found new importance in the treatment of allergy to chemotherapy and biologics. Not only does this help patients with cancer, but also patients who use biologics for a variety of connective tissue disorders, inflammatory bowel disease, and vasculitis. Desensitization is highly effective in treating immediate hypersensitivity reactions regardless of whether the reaction is $\operatorname{IgE}$ or non-IgE mediated. When executed properly, it is highly successful irrespective of the initial grade of anaphlyaxis. This is significant since "treating through" high grade anaphylactic reactions with slow infusion and/or maximal premedication regimens could lead to serious clinical consequences and is not recommended. By keeping patients on first-line therapy, better clinical outcomes and fewer complications from treatment failures are achieved, thereby reducing mortality, morbidity, and healthcare spending. There is typically no sacrifice

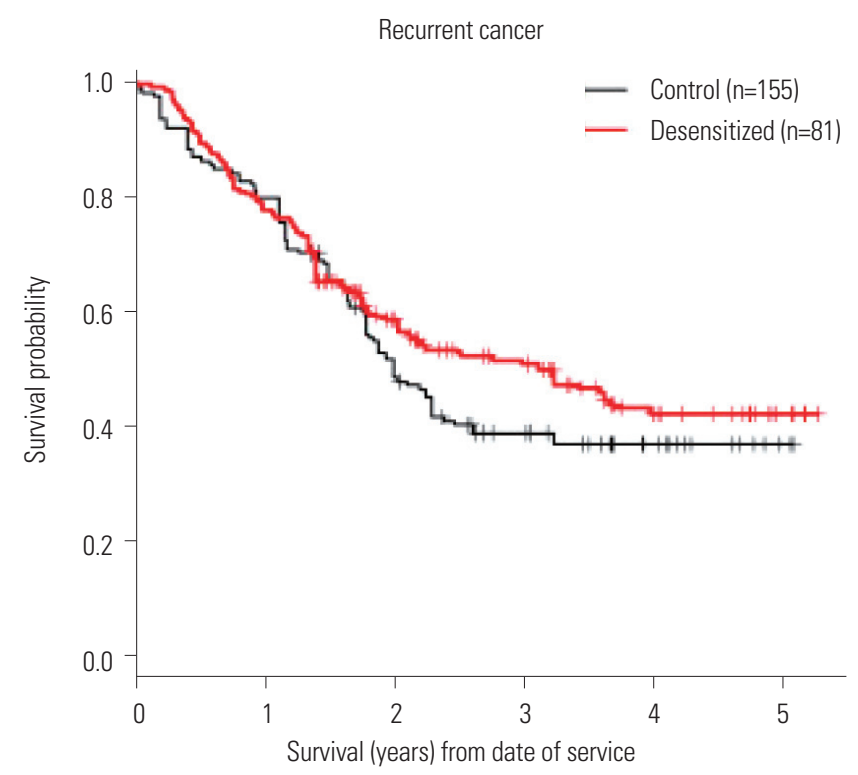

Fig. 4. Kaplan-Meier curve comparing surivival of patients receiving carboplatin by desensitization (red) vs. normal infusion (black). Adapted from Sloane, et al. J Allergy Clin Immunol Pract 2016;4:497-504, with permission of Elsevier [32]. in drug efficacy when administered via desensitization, and since the early studies on chemotherapy desensitization were published in the early 2000s, other groups have described other desensitization protocols which are variations on the theme of gradual dose uptitration from a very low starting dose. These newer protocols seem to show similar safety and efficacy as the protocol used at BWH, but use different numbers of steps, dilutions, or both to successfully desensitize patients. ${ }^{33-36}$ Having an allergist screen patients for and supervise desensitizations is highly recommended since their expertise can 1) help risk-stratify the patients based on clinical historytaking and skin testing and 2) recognize and treat potentially dangerous anaphylactic breakthrough reactions in a timely manner. The question of whether desensitization is appropriate is not always clear, and because desensitization may require special resources which are potentially limited (dedicated nursing, longer time occupying an infusion bed, special pharmacy expertise), it should be used only when necessary. The taxane treatment algorithm described earlier by Picard, et al. ${ }^{27}$ is an especially useful tool in this regard. Severe breakthrough reactions do sometimes occur and having an allergy specialist knowledgeable in the treatment of anaphylaxis is a critically important safety measure. A cautionary note can be taken from the experience of the only known published case of a patient death during desensitization. In this case, a patient with previous tolerance to carboplatin desensitization and known pulmonary hypertension died of cardiopulmonary arrest during an anaphylactic reaction in the last step of a carboplatin desensitization using a rapid 4-bag, 4-step desensitization protocol. ${ }^{37}$ While the specific circumstances of this patient's death were not described, this was a patient with poor baseline cardiopulmonary reserve, and illustrates why such patients should be approached with especially high caution. It also illustrates the danger of complacency in lowering vigilance to the risk that is never totally eliminated with desensitization. Early recognition and intervention is essential to any anaphylaxis management plan. ${ }^{38}$ Though desensitization does not eliminate the risk of hypersensitivity reactions, it is a powerful de-risking tool in the proper hands. The potential benefits of increasing patient access to desensitization are immense and should be the standard of care in institutions where it can be made available.

\section{ORCID iD}

David In-Chull Hong ～https://orcid.org/0000-0003-4701-8710

\section{REFERENCES}

1. Brown SG, Stone SF, Fatovich DM, Burrows SA, Holdgate A, Celenza A, et al. Anaphylaxis: clinical patterns, mediator release, and severity. J Allergy Clin Immunol 2013;132:1141-9.

2. Lenz HJ. Management and preparedness for infusion and hypersensitivity reactions. Oncologist 2007;12:601-9. 
3. Brown SG. Clinical features and severity grading of anaphylaxis. J Allergy Clin Immunol 2004;114:371-6.

4. O'Donovan WJ, Klorfajn I. Sensitivity to penicillin; anaphylaxis and desensitisation. Lancet 1946;2:444-6.

5. Peck SM, Siegal S, Bergamini R. Successful desensitization in penicillin sensitivity. J Am Med Assoc 1947;134:1546.

6. Gorman SK, Zed PJ, Dhingra VK, Ronco JJ. Rapid imipenem/ cilastatin desensitization for multidrug-resistant Acinetobacter pneumonia. Ann Pharmacother 2003;37:513-6.

7. Papakonstantinou G, Bogner JR, Hofmeister F, Hehlmann R. Cefotaxime desensitization. Clin Investig 1993;71:165-7.

8. Lantner RR. Ciprofloxacin desensitization in a patient with cystic fibrosis. J Allergy Clin Immunol 1995;96(6 Pt 1):1001-2.

9. Ozols RF, Bundy BN, Greer BE, Fowler JM, Clarke-Pearson D, Burger RA, et al. Phase III trial of carboplatin and paclitaxel compared with cisplatin and paclitaxel in patients with optimally resected stage III ovarian cancer: a Gynecologic Oncology Group study. J Clin Oncol 2003;21:3194-200.

10. du Bois A, Lück HJ, Meier W, Adams HP, Möbus V, Costa S, et al. A randomized clinical trial of cisplatin/paclitaxel versus carboplatin/paclitaxel as first-line treatment of ovarian cancer. J Natl Cancer Inst 2003;95:1320-9.

11. Lengyel E. Ovarian cancer development and metastasis. Am J Pathol 2010;177:1053-64.

12. Markman M, Kennedy A, Webster K, Elson P, Peterson G, Kulp B, et al. Clinical features of hypersensitivity reactions to carboplatin. J Clin Oncol 1999;17:1141.

13. Markman M, Zanotti K, Peterson G, Kulp B, Webster K, Belinson J. Expanded experience with an intradermal skin test to predict for the presence or absence of carboplatin hypersensitivity. J Clin Oncol 2003;21:4611-4.

14. Koshiba H, Hosokawa K, Kubo A, Miyagi Y, Oda T, Miyagi Y, et al. Incidence of carboplatin-related hypersensitivity reactions in Japanese patients with gynecologic malignancies. Int J Gynecol Cancer 2009;19:460-5.

15. Lax T, Long A, Banerji A. Skin testing in the evaluation and management of carboplatin-related hypersensitivity reactions. J Allergy Clin Immunol Pract 2015;3:856-62.

16. Weiss RB, Donehower RC, Wiernik PH, Ohnuma T, Gralla RJ, Trump DL, et al. Hypersensitivity reactions from taxol. J Clin Oncol 1990;8:1263-8.

17. Markman M, Kennedy A, Webster K, Kulp B, Peterson G, Belinson J. Paclitaxel-associated hypersensitivity reactions: experience of the gynecologic oncology program of the Cleveland Clinic Cancer Center. J Clin Oncol 2000;18:102-5.

18. Lee CW, Matulonis UA, Castells MC. Carboplatin hypersensitivity: a 6-h 12-step protocol effective in 35 desensitizations in patients with gynecological malignancies and mast cell/IgE-mediated reactions. Gynecol Oncol 2004;95:370-6.

19. Castells MC, Tennant NM, Sloane DE, Hsu FI, Barrett NA, Hong DI, et al. Hypersensitivity reactions to chemotherapy: outcomes and safety of rapid desensitization in 413 cases. J Allergy Clin Immunol 2008;122:574-80.

20. Feldweg AM, Lee CW, Matulonis UA, Castells M. Rapid desensitization for hypersensitivity reactions to paclitaxel and docetaxel: a new standard protocol used in 77 successful treatments. Gynecol Oncol 2005;96:824-9.

21. Brennan PJ, Rodriguez Bouza T, Hsu FI, Sloane DE, Castells MC. Hypersensitivity reactions to mAbs: 105 desensitizations in 23 patients, from evaluation to treatment. J Allergy Clin Immunol 2009;124:1259-66.

22. Dye D, Watkins J. Suspected anaphylactic reaction to Cremophor
EL. Br Med J 1980;280:1353.

23. Lorenz W, Reimann HJ, Schmal A, Dormann P, Schwarz B, Neugebauer E, et al. Histamine release in dogs by Cremophor E1 and its derivatives: oxethylated oleic acid is the most effective constituent. Agents Actions 1977;7:63-7.

24. Weiszhár Z, Czúcz J, Révész C, Rosivall L, Szebeni J, Rozsnyay Z. Complement activation by polyethoxylated pharmaceutical surfactants: Cremophor-EL, Tween-80 and Tween-20. Eur J Pharm Sci 2012;45:492-8.

25. Hüttel MS, Schou Olesen A, Stoffersen E. Complement-mediated reactions to diazepam with Cremophor as solvent (Stesolid MR). Br J Anaesth 1980;52:77-9.

26. Fader AN, Rose PG. Abraxane for the treatment of gynecologic cancer patients with severe hypersensitivity reactions to paclitaxel. Int J Gynecol Cancer 2009;19:1281-3.

27. Picard M, Pur L, Caiado J, Giavina-Bianchi P, Galvão VR, Berlin ST, et al. Risk stratification and skin testing to guide re-exposure in taxane-induced hypersensitivity reactions. J Allergy Clin Immunol 2016;137:1154-64.

28. Ha E, Wang W, Wang YJ. Peroxide formation in polysorbate 80 and protein stability. J Pharm Sci 2002;91:2252-64.

29. Commins SP, Platts-Mills TA. Anaphylaxis syndromes related to a new mammalian cross-reactive carbohydrate determinant. J Allergy Clin Immunol 2009;124:652-7.

30. Commins SP, James HR, Kelly LA, Pochan SL, Workman LJ, Perzanowski MS, et al. The relevance of tick bites to the production of IgE antibodies to the mammalian oligosaccharide galactose- $\alpha$ 1,3-galactose. J Allergy Clin Immunol 2011;127:1286-93.

31. Chung CH, Mirakhur B, Chan E, Le QT, Berlin J, Morse M, et al. Cetuximab-induced anaphylaxis and IgE specific for galactosealpha-1,3-galactose. N Engl J Med 2008;358:1109-17.

32. Sloane D, Govindarajulu U, Harrow-Mortelliti J, Barry W, Hsu FI, Hong D, et al. Safety, costs, and efficacy of rapid drug desensitizations to chemotherapy and monoclonal antibodies. J Allergy Clin Immunol Pract 2016;4:497-504.

33. Gastaminza G, de la Borbolla JM, Goikoetxea MJ, Escudero R, Antón J, Espinós J, et al. A new rapid desensitization protocol for chemotherapy agents. J Investig Allergol Clin Immunol 2011;21: 108-12.

34. Madrigal-Burgaleta R, Berges-Gimeno MP, Angel-Pereira D, Ferreiro-Monteagudo R, Guillen-Ponce C, Pueyo C, et al. Hypersensitivity and desensitization to antineoplastic agents: outcomes of 189 procedures with a new short protocol and novel diagnostic tools assessment. Allergy 2013;68:853-61.

35. Madrigal-Burgaleta R, Bernal-Rubio L, Berges-Gimeno MP, Carpio-Escalona LV, Gehlhaar P, Alvarez-Cuesta E. A large singlehospital experience using drug provocation testing and rapid drug desensitization in hypersensitivity to antineoplastic and biological agents. J Allergy Clin Immunol Pract 2018 Aug 8 [Epub]. Available at: https://doi.org/10.1016/j.jaip.2018.07.031.

36. Takase N, Matsumoto K, Onoe T, Kitao A, Tanioka M, Kikukawa Y, et al. 4-step 4-h carboplatin desensitization protocol for patients with gynecological malignancies showing platinum hypersensitivity: a retrospective study. Int J Clin Oncol 2015;20:566-73.

37. Altwerger G, Gressel GM, English DP, Nelson WK, Carusillo N, Silasi DA, et al. Platinum desensitization in patients with carboplatin hypersensitivity: a single-institution retrospective study. Gynecol Oncol 2017;144:77-82.

38. Lieberman P, Nicklas RA, Randolph C, Oppenheimer J, Bernstein D, Bernstein J, et al. Anaphylaxis--a practice parameter update 2015. Ann Allergy Asthma Immunol 2015;115:341-84. 